\title{
Práticas Educativas e Indicadores de Ansiedade, Depressão e Estresse Maternos
}

\author{
Olga Maria Piazentin Rolim Rodrigues ${ }^{1}$ \\ Sária Cristina Nogueira \\ Universidade Estadual Paulista - UNESP Bauru
}

\begin{abstract}
RESUMO - Compararam-se práticas educativas parentais positivas e negativas de 100 mães de bebês, com e sem indicadores de ansiedade, depressão e estresse. Utilizou-se o Inventário de Estilos Parentais de Mães de Bebês, o BDI-III, o IDATE e o ISSL. Com o Teste $\mathrm{t}$ de Student, compararam-se os grupos relativamente à presença/ausência de cada indicador e à quantidade de indicadores presentes. Considerou-se como grupo controle as mães sem nenhum dos indicadores emocionais avaliados. Observou-se a presença das práticas negativas punição inconsistente e disciplina relaxada em todos os indicadores avaliados. A presença de dois ou mais indicadores aumentou a frequência da prática negativa de punição inconsistente. Identificar o tipo de prática educativa presente pode contribuir para intervenções pontuais mais eficientes.
\end{abstract}

Palavras-chave: estilo parental, práticas educativas, relações mãe-criança, depressão, ansiedade, stress

\section{Educational Practices and Indicartors of Anxiety, Depression and Maternal Stress}

\begin{abstract}
Positive and negative parenting educational practices of 100 mothers of babies with and without indicators of anxiety, depression and stress were compared. In this study the Inventory of Parental Styles of Baby's Mothers, the BDIIII, STAI and ISSL were administered. Groups with and without the presence of indicators and with different amounts of indicators were compared using the Student's t-test. Mothers without any of the evaluated emotional indicators were considered as a control group. The presence of the negative practices Inconsistent Punishment and Relaxed Discipline was observed in all evaluated indicators. The results indicate that the presence of two or more increased the frequency of negative practice Inconsistent Punishment. Identifying the type of educational practices can contribute to more efficient punctual interventions.
\end{abstract}

Keywords: parenting style, childrearing practices, mother-child relations, depression, anxiety, stress

As formas de interação dos pais com seus filhos podem promover comportamentos socialmente adequados, assim como favorecer a emergência e manutenção de comportamentos socialmente inadequados (Bolsoni-Silva \& Marturano, 2007). Na criação de seus filhos, os pais utilizam práticas educativas parentais, cujo objetivo é educar, socializar e controlar seus comportamentos (Patterson, Reid, \& Dishion, 2002). Para serem contingentes, os pais devem consequenciar os comportamentos dos filhos imediatamente após a ocorrência desses comportamentos e não de acordo com outras variáveis, como o seu próprio humor, por exemplo (Gomide, 2006). Quando há efetividade e consistência nas práticas parentais e os pais são responsivos às necessidades dos filhos, favorece-se a baixa frequência ou a redução dos comportamentos inadequados (agressividade, desrespeito às regras, birra etc.) e a ocorrência de comportamentos socialmente adequados (Bolsoni-Silva \& Marturano, 2007). Bolsoni-Silva e Marturano descrevem que comportamentos adequados ou inadequados estão relacionados às expectativas culturais, sendo que os primeiros são aqueles que facilitam interações sociais e aprendizagens significativas da criança, ao contrário dos comportamentos do segundo tipo.

Considerando a importância das práticas educativas parentais para o desenvolvimento de repertórios infantis

1 Endereço para correspondência: Departamento de Psicologia, Universidade Estadual de São Paulo, Bauru, UNESP, Av. Eng ${ }^{\circ}$ Luiz Edmundo Carrijo Coube, $\mathrm{n}^{\circ}$ 14-01, Vargem Limpa, Bauru-SP, Brasil. CEP: 17.033-360.E-mail: olgarolim@fc.unesp.br adequados ou inadequados, Altafim (2012) chama a atenção para a importância de identificar o tipo de prática que pais e mães de bebês estão utilizando para educá-los. A autora ressalta que as práticas negativas já são identificadas no repertório comportamental de mães de bebês com idade até 12 meses, de maneira que as intervenções precoces preventivas podem ser propícias para que sejam instaladas práticas positivas e minimizadas ou eliminadas práticas negativas. É importante identificar as práticas educativas parentais (positivas e negativas) e os fatores de risco para o seu desenvolvimento em pais de bebês - entre eles, os indicadores emocionais -, considerando que ações preventivas podem contribuir para evitar futuros problemas de comportamento infantil (Altafim, 2012; Ferreira \& Marturano, 2002).

Gomide (2006) apresenta um modelo teórico no qual descreve sete práticas educativas parentais. Dessas, duas são consideradas positivas: a monitoria positiva, que se refere à atenção para a localização dos filhos, para a qualidade das atividades por eles desenvolvidas, bem como à promoção da adaptação dos mesmos aos diferentes contextos; e comportamento moral, que remete ao ensino de valores culturalmente aceitos. As cinco restantes são negativas, a saber: negligência, que pode ser entendida como a ausência de atenção e afeto; abuso físico e psicológico, que se refere ao uso de ameaças, chantagens ou castigos físicos; disciplina relaxada, que remete ao fato de os pais não cumprirem as regras pré-estabelecidas por eles próprios; punição inconsistente, que consiste na dependência do humor dos pais para que esses punam ou reforcem os comportamentos 
de seus filhos; e, por fim, monitoria negativa, que se refere a um excesso de regras, resultando em seu não cumprimento e em um clima de hostilidade.

O conjunto de práticas educativas parentais utilizadas pelos pais ou cuidadores é definido como estilo parental (Gomide, Salvo, Pinheiro, \& Sabbag, 2005). A partir desse modelo, Gomide (2006) formulou o Inventário de Estilos Parentais, objetivando diagnosticar famílias de risco e de não risco social para o desenvolvimento infantil. Como o referido instrumento não se propõe a avaliar as práticas educativas de mães e pais de bebês, Altafim, Schiavo e Rodrigues (2008) adaptaram o instrumento e desenvolveram o Inventário de Estilos Parentais para Mães de Bebês (IEPMB). Rodrigues, Nogueira e Altafim (2013) avaliaram práticas educativa de mães de bebês de um a 22 meses de idade, assim como sexo e idade do bebê, tipo de família e número de filhos. Os resultados demonstraram que as mães utilizam menos práticas educativas positivas com os meninos, mais negligência com os bebês mais velhos, mais punição inconsistente se a família é nuclear e mais abuso físico se têm mais de um filho. Comparando as práticas educativas maternas e considerando a idade dos filhos, Nogueira, Rodrigues e Altafim (2013) observaram que, independente da idade, as mães utilizam a prática de Monitoria Positiva. No entanto, entre as mães adolescentes (menos de 18 anos), identificaram alta frequência da prática de disciplina relaxada. Entre as mães adultas, os resultados apontaram para uso mais frequente das práticas educativas negativas de negligência e disciplina relaxada.

Para Belsky (1984), recursos psicológicos dos pais, características das crianças e contexto social em que estes vivem, incluindo as fontes de estresse e de suporte social, são fatores que poderiam influenciar o uso de práticas educativas parentais, o que torna o exercício da parentalidade multideterminado. Manuel, Martinson, Bledsoe-Mansori e Bellamy (2012) reforçam que a maternidade requer recursos financeiros e psicossociais e a escassez deles tem sido associada a grandes esforços pessoais, aumentando o risco de depressão materna e outros problemas de saúde mental, que, indiretamente, incidirão sobre o desenvolvimento dos bebês. Assim, uma vez que a literatura aponta para a influência das questões referentes à saúde mental nas práticas educativas parentais, parece importante identificar que tipo de prática está associado à presença de determinados indicadores emocionais presentes em mães de bebês.

Pereira et al. (2012) realizaram um estudo com 291 mães adultas de bebês de 16 meses, cujo objetivo era verificar se a história de maus-tratos maternos e o atual estresse parental estavam associados a dificuldades parentais. Os resultados apontaram que mães com relato de maus-tratos infantis apresentaram alto estresse parental $(0,29$, com $p<0,005)$ e baixa sensibilidade em relação a suas crianças $(0,13$, com $p<0,05)$. Tais dados demonstram a importância de considerar possíveis fatores de vulnerabilidade materna que podem influenciar as práticas educativas parentais emitidas, como as variáveis relacionadas à saúde mental das mães, dentre as quais se encontram a ansiedade, a depressão e o estresse.

Para a Análise do Comportamento, a ansiedade pode referir-se a eventos diversos, como os estados internos dos falantes e os processos comportamentais que os produzem.
Para Zamignani e Banaco (2005), a ansiedade consiste na emissão de respostas de fuga e esquiva com o objetivo de eliminar estímulos temidos e de respostas repetitivas que pospõem o estímulo aversivo. Enquanto fenômeno clínico, esses comportamentos de fuga e esquiva ocupam tempo considerável do dia dos indivíduos, impedindo o desempenho das atividades profissionais, sociais e acadêmicas e envolvendo grau de sofrimento considerado significativo.

Para Correia e Linhares (2007), há situações em que a ansiedade pode atuar como sinal de alerta, cooperando para a preservação da vida, ou seja, funcionando como propulsora para a emissão de comportamentos de enfrentamento diante de situações que resultam nesse estado ansioso. Todavia, alertam que as manifestações comportamentais de ansiedade podem exacerbar-se, atuando em nível desproporcional à situação que a desencadeou, podendo surgir sem motivo específico ou apresentando-se como resposta inadequada aos eventos estressores, caracterizando-se, então, como patológica.

Em um procedimento de avaliação dos níveis de ansiedade, há que se fazer a distinção entre: ansiedade-traço e ansiedade-estado. A primeira remete a uma característica estável do indivíduo, representando o modo como ele tende a reagir diante de situações estressantes ou conflituosas e a segunda representa uma condição emocional transitória caracterizada pelo aumento subjetivo da tensão em função de eventos considerados potencialmente críticos (Cury \& Menezes, 2006).

O nível de ansiedade materna pode ser caracterizado como fator de risco para o desenvolvimento dos filhos (Fraga, Linhares, Carvalho, \& Martinez, 2008), pois pode alterar a responsividade das mães diante do bebê (Perosa, Canavez, Silveira, Padovani, \& Peraçoli, 2009). Wijnroks (1999) destacou que mães ansiosas interagiam de forma mais ativa, mas se comportavam de modo mais intrusivo, emitindo comportamentos inadequados e sem sensibilidade, utilizando-se de super-estimulação e interferência nas atividades do bebê. Também Kaitz, Maytal, Devor, Bergman e Mankuta (2010) avaliaram a associação entre ansiedade materna, características de interação entre a mãe e seu bebê e a regulação das emoções frente a situações estressantes. Eles concluíram que o comportamento de ansiedade materna resulta na hipervigilância sobre os bebês. Os estudos em questão apontam para práticas positivas (interagem de forma mais ativa) e negativas (intrusividade, superestimulação e hipervigilância) o que mostra a importância de estudos que identifiquem o tipo de prática negativa presente no repertório materno quando indicadores emocionais estão presentes.

O estudo de Bekkhus, Rutter, Barker e Borge (2011) examinou as relações entre riscos familiares, incluindo a desarmonia do casal, bem como ansiedade e depressão maternas durante os períodos pré e pós-natal, associando-os a comportamentos de choro e agressão física das crianças quando estas tinham 36 meses de idade. Os resultados mostraram associação positiva entre os comportamentos de choro e de agressão física das crianças e os níveis de ansiedade e depressão materna, assim como de desarmonia do casal.

Ferster, Culbertson, Boren e Perrot (1977) descrevem a depressão como diminuição dos comportamentos 
positivamente reforçados e aumento da frequência de comportamentos de fuga e esquiva de estímulos aversivos, o que ocorre na interação do indivíduo com seu ambiente. O termo disforia é utilizado para designar sentimentos referentes à tristeza e infelicidade (Levinsohn, 1976, citado por Gongora, 1981). Assim, o humor bastante rebaixado pode ser classificado como humor disfórico e, uma vez que os distúrbios de humor são acentuados, pode-se definir a depressão como humor disfórico (Gongora, 1981). Vale ressaltar que, para Gongora, a utilização do conceito de disforia é bastante limitada para a descrição do fenômeno clínico de depressão, uma vez que esse engloba outras características relevantes e que a alteração do humor é apenas uma parte da síndrome depressiva.

Field (1992, 1995) aponta que as mães com humor depressivo podem não oferecer ao bebê a estimulação necessária a um desenvolvimento adequado, podendo os filhos tornarem-se, também, deprimidos, com baixo nível de atividade e embotamento afetivo, além de não desenvolverem as habilidades necessárias para interações sociais futuras. A autora também relata que os bebês de mães deprimidas podem desenvolver um estilo de humor deprimido nos primeiros três meses de vida, o que pode estender-se até o primeiro ano de vida caso a depressão materna persista e acarretar atrasos no desenvolvimento e crescimento do bebê. Porém, identificar a influência da depressão sobre práticas educativas maternas especificas auxilia a elaboração de intervenções mais eficazes, sugerindo que o foco seja não só a depressão, mas a melhoria das suas práticas educativas negativas.

Um estudo longitudinal desenvolvido por Letourneau, Tramonte e Willms (2013) investigou a relação entre sintomas depressivos maternos e desenvolvimento infantil nas áreas cognitiva e comportamental, concluindo que os filhos de mães que apresentam sintomas depressivos têm aumento do risco de apresentar um baixo vocabulário receptivo, desatenção e agressividade nas idades de quatro e cinco anos. Esses autores consideraram que esses achados estão parcialmente relacionados a fatores demográficos, funcionamento familiar e parentalidade. Segundo os autores, crianças expostas aos sintomas depressivos maternos aos dois e três anos de idade podem apresentar ansiedade no final da idade escolar.

Em um artigo de revisão da literatura publicada no período entre 1998 e 2003, Correia e Linhares (2007) constataram que a ansiedade e a depressão materna podem afetar o desenvolvimento infantil, uma vez que a criança está exposta ao sofrimento dos pais. As autoras identificaram a ocorrência concomitante de ansiedade e depressão materna, independentemente da condição de nascimento do bebê e do momento de avaliação (pré ou pós-natal). Além disso, evidenciou-se que mães são mais vulneráveis que os pais, apresentando maior nível de ansiedade, o que corrobora a ideia de que as mulheres estão mais propensas ao comprometimento da saúde mental devido às mudanças decorrentes da gravidez, parto e cuidados com o bebê.

Considerando o primeiro ano de vida do bebê como um período sensível, Bagner, Pettit, Lewinsohn e Seeley (2010) examinaram os efeitos da depressão materna durante esse período em problemas de comportamento subsequentes. Os autores concluíram que a presença da depressão no primeiro ano de vida do bebê representa aumento no risco de consequências adversas para ele, sugerindo a relevância da identificação, prevenção e intervenção precoce nesses casos.

Farmer e Lee (2011) conduziram um estudo longitudinal para avaliar a influência da depressão e do estresse sobre a interação mãe-bebê. Os resultados desse estudo revelaram que mães com estresse parental tendem a apresentar pouco senso de controle percebido, relatando experiência de depressão e provendo pouca estimulação cognitiva às crianças. Além disso, notou-se que mães que apresentavam alto senso de controle não relataram a experiência de depressão e proviam mais estimulação cognitiva às crianças.

Além da depressão materna, os níveis de estresse materno também têm sido apontados como fatores de risco para a interação das mães e seus bebês, marcadas pelas práticas educativas parentais. Banaco (2005) define o estresse como uma alteração na relação do indivíduo com o ambiente, devido a alterações ambientais aversivas, o que lhe demanda um novo repertório comportamental. Caso o indivíduo não apresente respostas comportamentais adaptativas, o estresse tende a agravar-se. O estresse pode ser entendido como uma expressão da reação de um organismo frente a situações difíceis ou excitantes, sendo essas provocadoras de alterações psicológicas, físicas e químicas, influenciando os comportamentos dos indivíduos (Gomes \& Bosa, 2004).

O estresse foi descrito por Selye (1956) em três fases: (a) fase de alerta, que se refere ao desequilíbrio homeostático que ocorre quando a pessoa se depara com um evento estressor, fazendo com que ela se prepare para uma "luta ou fuga". É uma reação que pode ser considerada benéfica à medida que prepara a pessoa para a ação em situações de urgência; (b) fase de resistência, quando a fase de alerta persiste e há longa duração ou grande intensidade do evento estressor, fazendo com que a pessoa utilize suas reservas de energia adaptativa, na tentativa de um reequilíbrio. Nesta fase, caso a reserva de energia adaptativa seja suficiente, ela pode se recuperar e abandonar o processo de estresse; (c) fase de exaustão, que ocorre quando a pessoa encontra-se esgotada pelo grande consumo de energia na tentativa de reestabelecer o equilíbrio homeostático. Esta fase tende a ocorrer quando sua resistência não foi suficiente para lidar com a fonte de estresse ou se houver, em concomitância, a presença de outros estressores (Camelo \& Angerami, 2004; Goulart Jr. \& Lipp, 2008). Lipp (2000) descreveu uma fase intermediária, entre a fase de resistência e a de exaustão, denominada de quaseexaustão. Essa fase caracteriza-se por um enfraquecimento da pessoa que não está conseguindo adaptar-se ou resistir ao evento estressor, mas que ainda não tenha atingido a exaustão completa.

É possível que as mulheres apresentem sintomas de estresse devido a transformações decorrentes do período da gestação, do nascimento do bebê, dos cuidados com o bebê e, no caso de muitas mães, do retorno ao trabalho. Uma vez que tais sintomas sejam identificados e compreendidos, podem ser objeto de intervenção com o objetivo de minimizar os seus efeitos sobre a saúde mental materna e, por consequência, sobre o desenvolvimento infantil. $O$ estresse parental influencia as práticas educativas dos pais, de maneira que os comportamentos emitidos por eles podem promover e 
aumentar a frequência de comportamentos inadequados da criança (Kazdin \& Whitley, 2003).

A literatura consultada acerca da influência dos indicadores emocionais maternos sobre as práticas educativas maternas sugere a ocorrência de comportamentos como hipervigilância, intrusividade, baixa sensibilidade aos filhos e pouca estimulação cognitiva (Farmer \& Lee, 2011; Kaitz et al., 2010; Pereira et al., 2012; Wijnroks, 1999). Outros estudos referem-se à influência de indicadores emocionais sobre o desenvolvimento da criança, relatando atrasos comportamentais ou a presença de comportamentos inadequados, como agressão e choro excessivo (Bekkhus et al., 2011; Field, 1992, 1995; Letourneau et al., 2013). Assim, pretendeu-se, no presente estudo, comparar as práticas parentais positivas e negativas de mães de bebês que têm ou não indicadores clínicos de ansiedade, depressão e estresse.

\section{Método}

\section{Participantes}

Participaram deste estudo 100 mães, com idade entre 15 a 39 anos (Média $=26,2 ; D P=5,91)$. Seus bebês tinham entre seis e 12 meses (Média $=8,7 ; D P=2,24$ ). Tais mães constituíram uma amostra de conveniência, uma vez que frequentavam o projeto de extensão Acompanhamento do desenvolvimento de bebês: Avaliação e orientação aos pais, que funciona em uma clínica escola em uma universidade do interior do estado de São Paulo. Esse projeto tem como objetivo monitorar o desenvolvimento de bebês até 12 meses, não prevendo nenhuma intervenção com as mães. Casos identificados no presente estudo foram encaminhados para o atendimento para adultos ou serviços da cidade.

\section{Local}

A coleta de dados foi realizada em sala para atendimento individual, em um Centro de Psicologia Aplicada (CPA) de uma universidade pública paulista. Essa sala contém uma mesa, duas cadeiras e uma poltrona, compondo o ambiente adequado para a coleta de dados individual.

\section{Instrumentos}

Para a avaliação das práticas educativas parentais, foi utilizado o IEPMB, adaptado de Gomide (2006) por Altafim et al. (2008). O IEPMB é composto por 25 itens, sendo cinco referentes a cada uma das práticas: monitoria positiva, negligência, disciplina relaxada, punição inconsistente e abuso físico. As respostas são dadas em uma escala likert de 3 pontos, em que a resposta "sempre" vale 2 pontos; "às vezes", 1 ponto; e "nunca", 0 (zero) ponto. Portanto, cada prática educativa pode ter a pontuação máxima de 10 pontos. Nas práticas educativas parentais negativas, quanto maior a pontuação, piores são as práticas parentais, já, para a prática monitoria positiva, quanto maior o escore, melhores são as práticas. O IEPMB tem sido utilizado em diversas pesquisas e tem se mostrado um instrumento eficiente e de fácil aplicação, para a identificação precoce de práticas parentais (Altafim et al., 2008; Nogueira et al., 2013; Rodrigues et al., 2011). Esse instrumento está em fase final de validação.

A ansiedade foi avaliada pelo Inventário de Ansiedade Traço-Estado (IDATE, 2003), que foi elaborado por Spielberger, Gorsuch e Lushene, tendo sido adaptado para o português por Biaggio. Trata-se de um instrumento de autoaplicação para jovens e adultos. O inventário é composto por duas escalas, elaboradas para medir dois conceitos distintos de ansiedade: estado de ansiedade (A-estado) e traço de ansiedade (T-traço). Utilizou-se o critério de corte (indicador em nível clínico) de escore igual ou acima do percentil 75 (ou escore 48) para definir sintomas de ansiedade clínica. Há, na literatura, estudos que utilizaram esse mesmo critério para definir ansiedade clínica (Padovani, Linhares, Carvalho, Duarte, \& Martinez, 2004; Perosa, Silveira, \& Canavez, 2008; Schiavo, 2011). As participantes que não atingiram escores acima do percentil 75 foram definidas como apresentando sintomas de ansiedade controlada, definição também já utilizada na literatura (Baptista, Baptista, \& Torres, 2006; Schiavo, 2011). O alfa de Cronbach nas duas escalas do IDATE revelaram índices de consistência altos, variando entre 0,82 e 0,89 (Fioravante, Santos, Maissonette, Cruz, \& Landeira-Fernandes, 2006).

A depressão foi avaliada pelo Inventário de Depressão de Beck (BDI-II), que consiste em uma escala de autorrelato de 21 itens, cada um com quatro alternativas, subentendendo graus crescentes ( 0 a 3) de gravidade da depressão (Gorestein, Wang, Argimon, \& Werlang, 2011). O objetivo desse instrumento é medir a intensidade da depressão em adultos e adolescentes a partir dos 13 anos de idade. O escore final pode variar de 0 a 63 . No presente estudo, utilizou-se o critério de indicador em nível clínico as pontuações maiores que 15 , indicativa de disforia, e maiores que 20, indicativa de depressão (Padovani et al., 2004; Perosa et al., 2008). Em termos de fidedignidade, encontrou-se um nível satisfatório de consistência interna (Coeficiente de Cronbach $=0,86$ ) e seu padrão de resposta é estável no tempo $(r=0,87 ; p<0,001)$ (Paranhos, Argemon, \& Werlang, 2010).

O estresse foi avaliado pelo Inventário de Sintomas de Stress para Adultos de Lipp (ISSL) (Lipp, 2000). O inventário, que se destina ao uso com jovens e adultos, é composto por três quadros, que se referem às quatro fases do estresse (Alerta, Resistência, Quase Exaustão e Exaustão), especificando os sintomas físicos e os psicológicos. Com as instruções do manual, os dados permitem que se identique: (a) se a pessoa apresenta sintomas significativos de estresse; (b) em que fase do estresse a pessoa se encontra; e (c) qual a sintomatologia mais presente: somática ou psicológica. No presente estudo, considerou-se como indicador clínico se a pessoa tem estresse, independente da pontuação e, consequentemente, da gravidade do caso. $\mathrm{O}$ instrumento apresenta bons indicadores de consistência interna (coeficiente alfa de Cronbach de 0,91) (Lipp, 2000) 


\section{Procedimento de coleta de dados}

Durante a participação das mães no projeto de extensão Acompanhamento do desenvolvimento de bebês: Avaliação e orientação aos pais, em qualquer dos meses, elas eram convidadas a participarem da presente pesquisa. Em caso de aceite, previamente se combinava um horário para a aplicação individual dos instrumentos na seguinte sequência: ISSL, IDATE, BDI-II e IEPMB. Optou-se por deixar o IEPMB por último para que, depois de responder questões sobre os próprios indicadores emocionais, as mães pudessem voltar-se para as questões relativas ao bebê e finalizar falando desse assunto. As questões de exemplos dos instrumentos eram feitas junto com o aplicador, que depois permanecia na sala enquanto as mães respondiam para resolver dúvidas, caso houvesse. Nesse caso, o aplicador as resolvia, procurando não interferir na resposta.

\section{Considerações éticas}

O presente estudo foi aprovado por comitê de ética em pesquisa de uma universidade pública do estado de São Paulo (processo $\mathrm{n}^{\circ} 4205 / 46 / 01 / 11$ ) e seguiu as diretrizes da Resolução no 446 (2012), do Conselho Nacional de Saúde - CNS. Ao aceitarem participar da pesquisa, as mães receberam todas as informações pertinentes ao projeto em relação aos horários e atividades realizadas, sendo informadas sobre o sigilo das informações por elas fornecidas quando da apresentação dos dados desta pesquisa em eventos e publicações da área. Foram esclarecidas, também, quanto à ausência de qualquer ônus para a participação na pesquisa, assim como da manutenção dos demais serviços por ela usufruídos na clínica-escola, em caso de desistência, o que poderia ocorrer em qualquer fase deste projeto. A partir do aceite e esclarecidas todas as dúvidas, as participantes assinaram um Termo de Consentimento Livre e Esclarecido.

\section{Análise dos dados}

Os dados foram analisados utilizando o pacote estatístico SPSS 17.0 (Statistical Package for the Social Sciences). Foram verificadas as comparações entre práticas educativas parentais e níveis de ansiedade, estresse e depressão maternos. Para tanto, foi utilizado o Teste $t$ de Student para amostras independentes, para avaliar a existência de diferença significativa entre as médias dos postos de duas condições (Dancey \& Reidy, 2006). Utilizou-se como critério de significância $p<0,05$.

Inicialmente as participantes foram divididas em grupos considerando a presença e ausência de cada um dos indicadores emocionais e suas práticas educativas foram comparadas. Em seguida, elas foram divididas em quatro grupos de acordo com a presença (ou não) dos indicadores emocionais: o Grupo 1 era composto por 25 mães que não apresentaram indicadores emocionais para depressão, estresse ou ansiedade; o Grupo 2 era composto por 25 mães que apresentaram apenas um indicador; o Grupo 3 era formado por 33 mães que apresentaram pelo menos dois indicadores emocionais (estresse e ansiedade, estresse e depressão, ansiedade e depressão); e o Grupo 4 era composto por 16 mães que apresentaram todos os indicadores em nível clínico. As comparações foram feitas entre os Grupos 1 e 2; 1 e 3 e 1 e 4 . A hipótese que norteou o presente estudo é de que, quanto mais indicadores, maiores os índices de práticas educativas parentais negativas.

\section{Resultados}

Os resultados se referem, inicialmente, à comparação das práticas educativas maternas, considerando a presença e a ausência de cada um dos indicadores emocionais. As participantes sem estresse eram 32 e 68 , com estresse. Os grupos eram equivalentes com relação à idade $(p=0,896)$ e à escolaridade $(p=0,099)$. Observou-se diferença significativa em disciplina relaxada $(p=0,033)$, com média maior para o grupo com estresse (Tabela 1). Das participantes em ansiedade-estado, 66 não apresentaram indicadores clínicos. Os grupos com e sem ansiedade-estado eram semelhantes em escolaridade $(p=0,095)$ e em idade $(p=0,920)$. Observou-se diferença significativa em punição inconsistente $(p=0,018)$, com média maior para o grupo com ansiedade-estado. Delas, 54 não apresentaram indicadores clínicos para ansiedade-traço. Os grupos com e sem ansiedade-traço eram semelhantes em idade $(p=0,142)$, mas não em escolaridade $(p=0,001)$, que era mais baixa entre as mães com ansiedadetraço. Observou-se diferença significativa em disciplina relaxada $(p=0,024)$ e em punição inconsistente $(p=0,002)$. Nos dois casos, a média maior foi das mães com ansiedadetraço. Considerando a depressão, ela esteve presente em 29 das participantes. Os grupos com e sem depressão eram semelhantes em escolaridade $(p=0,067)$ e em idade $(p=0,744)$. Observou-se diferença estatisticamente significativa em punição inconsistente $(p=0,009)$, com média maior entre as mães com depressão (Tabela 1). A prática disciplina relaxada refere-se a comportamentos maternos inconsistentes como, por exemplo, ceder ao choro do bebê pegando-o no colo, depois de dizer que não o pegaria porque sabe que é manha; não manter uma rotina de sono, alimentação, banho. A prática negativa de punição inconsistente refere-se a mães que agem em função do humor nos cuidados dispensados ao filho. Por exemplo, acaricia-o ou atende às suas necessidades quando está bem e ignora-o quando está mal.

Outra análise realizada teve como objetivo avaliar se a quantidade de indicadores tinha algum impacto nas práticas educativas parentais. A Tabela 2 mostra esses dados. O Grupo 1 era composto por 25 mães sem indicadores e o Grupo 2 era composto por 25 mães com um indicador, sendo que $80 \%$ delas tinham somente estresse. Os grupos eram semelhantes em idade $(p=0,851)$ e escolaridade $(p=1,00)$. Não se observaram diferenças estatisticamente significativas entre os dois grupos em nenhuma das práticas analisadas. O Grupo 3 era composto por 33 mães que tinham dois ou três indicadores emocionais. Optou-se por juntá-las devido ao pequeno número de mães com apenas dois indicadores $(18 \%)$. Os Grupos 1 e 3 eram semelhantes em idade $(p=0,568)$ e em escolaridade $(p=0,104)$. Observaram-se diferenças estatisticamente significativas em punição inconsistente 
( $p=0,033)$, com frequência maior para as mães com dois ou três indicadores emocionais. O Grupo 4 era composto por 16 mães com todos os indicadores emocionais em nível clínico. Comparando-as com as mães do Grupo 1, eram semelhantes em idade ( $p=0863)$, mas não em escolaridade $(p=0,023)$, sendo que as mães do Grupo 4 tinham escolaridade menor. Observou-se diferença estatisticamente significativa em punição inconsistente $(p=0,002)$, com média maior para as mães do Grupo 4.

\section{Discussão}

O presente estudo pretendeu avaliar a influência da saúde mental materna sobre as práticas educativas parentais. A análise dessas práticas apontou que as mães apresentaram uma média alta da prática de monitoria positiva, que se refere essencialmente à vigilância e à responsividade materna em relação ao filho. Tais dados já foram observados em outros estudos com mães de bebês (Altafim et al., 2008; Nogueira et al., 2013; Rodrigues et al., 2011; Rodrigues et al., 2013). A presença de monitoria positiva tem sido considerada um fator de proteção, mesmo quando estão presentes práticas negativas (Gomide et al., 2005).

Para Salvo, Silvares e Toni (2005), o uso da monitoria positiva é preditor da sociabilidade infantil, ao passo que seu pouco uso prediz comportamentos de agressividade da criança. Parece, portanto, ser relevante que as mães de bebês façam uso dessa prática, acompanhando a criança em suas atividades, demonstrando afeto e contato físico com seus filhos, a fim de promover uma relação de confiança com eles desde tenra idade.

No entanto, para que possam exercer essa prática adequadamente, faz-se necessário que as mães também utilizem menos práticas negativas (Gomide, 2006). Os

Tabela 1. Comparação das médias das práticas educativas maternas considerando presença e ausência de indicadores emocionais de ansiedade, depressão e estresse.

\begin{tabular}{|c|c|c|c|c|c|c|c|c|c|c|}
\hline \multirow[t]{2}{*}{ Grupos } & \multicolumn{2}{|c|}{$\begin{array}{c}\text { Monitoria } \\
\text { positiva }\end{array}$} & \multicolumn{2}{|c|}{$\begin{array}{c}\text { Punição } \\
\text { inconsistente }\end{array}$} & \multicolumn{2}{|c|}{$\begin{array}{c}\text { Disciplina } \\
\text { relaxada }\end{array}$} & \multicolumn{2}{|c|}{ Negligência } & \multicolumn{2}{|c|}{$\begin{array}{c}\text { Abuso } \\
\text { físico }\end{array}$} \\
\hline & Média & DP & Média & DP & Média & $\mathrm{DP}$ & Média & DP & Média & DP \\
\hline Sem estresse $(n=32)$ & 9,0 & 1,0 & 1,56 & 1,68 & 3,65 & 2,47 & 1,31 & 1,42 & 0,34 & 0,86 \\
\hline Com estresse $(n=68)$ & 8,89 & 1,60 & 2,22 & 1,77 & 4,76 & 2,34 & 1,38 & 1,26 & 0,25 & 0,63 \\
\hline Valor de $p$ & \multicolumn{2}{|c|}{0,531} & \multicolumn{2}{|c|}{0,082} & \multicolumn{2}{|c|}{$\mathbf{0 , 0 3 3}$} & \multicolumn{2}{|c|}{0,806} & \multicolumn{2}{|c|}{0,542} \\
\hline Sem Ansiedade Estado $(n=66)$ & 8,87 & 1,49 & 1,71 & 1,31 & 4,31 & 2,28 & 1,30 & 1,30 & 0,22 & 0,54 \\
\hline Com Ansiedade Estado $(n=34)$ & 9,11 & 1,38 & 2,58 & 1,79 & 4,58 & 2,72 & 1,47 & 1,35 & 0,38 & 0,95 \\
\hline Valor de $p$ & \multicolumn{2}{|c|}{0,440} & \multicolumn{2}{|c|}{0,018} & \multicolumn{2}{|c|}{0,601} & \multicolumn{2}{|c|}{0,549} & \multicolumn{2}{|c|}{0,304} \\
\hline Sem Ansiedade Traço $(n=54)$ & 9,11 & 1,42 & 1,51 & 1,48 & 3,90 & 2,21 & 1,45 & 1,35 & 0,24 & 0,69 \\
\hline Com Ansiedade Traço $(n=46)$ & 8,78 & 1,48 & 2,58 & 1,90 & 5,00 & 2,55 & 1,28 & 1,27 & 0,32 & 0,73 \\
\hline Valor de $p$ & \multicolumn{2}{|c|}{0,263} & \multicolumn{2}{|c|}{0,002} & \multicolumn{2}{|c|}{$\mathbf{0 , 0 2 4}$} & \multicolumn{2}{|c|}{0,589} & \multicolumn{2}{|c|}{0,553} \\
\hline Sem Depressão $(n=71)$ & 9,01 & 1,42 & 1,71 & 1,56 & 4,30 & 2,33 & 1,23 & 1,27 & 0,26 & 0,69 \\
\hline Com Depressão $(n=29)$ & 8,82 & 1,53 & 2,72 & 2,03 & 4,65 & 2,66 & 1,65 & 1,39 & 0,31 & 0,76 \\
\hline Valor de $p$ & \multicolumn{2}{|c|}{0,564} & \multicolumn{2}{|c|}{0,009} & \multicolumn{2}{|c|}{0,522} & \multicolumn{2}{|c|}{0,152} & \multicolumn{2}{|c|}{0,787} \\
\hline
\end{tabular}

Tabela 2. Comparação das médias das práticas educativas maternas, considerando a quantidade de indicadores emocionais e a ausência deles.

\begin{tabular}{|c|c|c|c|c|c|c|c|c|c|c|}
\hline \multirow[t]{2}{*}{ Grupos } & \multicolumn{2}{|c|}{$\begin{array}{c}\text { Monitoria } \\
\text { positiva }\end{array}$} & \multicolumn{2}{|c|}{$\begin{array}{c}\text { Punição } \\
\text { inconsistente }\end{array}$} & \multicolumn{2}{|c|}{$\begin{array}{l}\text { Disciplina } \\
\text { relaxada }\end{array}$} & \multicolumn{2}{|c|}{ Negligência } & \multicolumn{2}{|c|}{$\begin{array}{c}\text { Abuso } \\
\text { físico }\end{array}$} \\
\hline & Média & DP & Média & DP & Média & DP & Média & DP & Média & DP \\
\hline G1 $(n=25)$ & 9,2 & 1,0 & 1,32 & 1,31 & 3,68 & 2,41 & 1,24 & 1,36 & 0,20 & 0,10 \\
\hline $\mathrm{G} 2(\mathrm{n}=25)$ & 8,9 & 1,84 & 1,72 & 1,56 & 4,4 & 2,02 & 1,36 & 1,28 & 0,32 & 0,18 \\
\hline Valor de $p$ & \multicolumn{2}{|c|}{0,508} & \multicolumn{2}{|c|}{0,333} & \multicolumn{2}{|c|}{0,258} & \multicolumn{2}{|c|}{0,750} & \multicolumn{2}{|c|}{0,563} \\
\hline G1 $(n=25)$ & 9,20 & 1,00 & 1,32 & 1,31 & 3,68 & 2,41 & 1,24 & 1,36 & 0,20 & 0,10 \\
\hline G3 $(n=33)$ & 8,84 & 1,27 & 2,21 & 1,79 & 4,72 & 2,41 & 1,45 & 1,41 & 0,24 & 0,61 \\
\hline Valor de $p$ & \multicolumn{2}{|c|}{0,261} & \multicolumn{2}{|c|}{$\mathbf{0 , 0 3 3}$} & \multicolumn{2}{|c|}{0,107} & \multicolumn{2}{|c|}{0,564} & \multicolumn{2}{|c|}{0,779} \\
\hline G1 $(n=25)$ & 9,2 & 1,0 & 1,32 & 1,31 & 3,68 & 2,41 & 1,24 & 1,36 & 0,20 & 0,10 \\
\hline $\mathrm{G} 4(\mathrm{n}=16)$ & 8,81 & 1,79 & 3,15 & 2,15 & 5,06 & 2,95 & 1,43 & 1,15 & 0,43 & 0,89 \\
\hline Valor de $p$ & \multicolumn{2}{|c|}{0,380} & \multicolumn{2}{|c|}{0,002} & \multicolumn{2}{|c|}{0,109} & \multicolumn{2}{|c|}{0,634} & \multicolumn{2}{|c|}{0,281} \\
\hline
\end{tabular}


dados obtidos apontaram a presença das práticas de punição inconsistente (humor dos pais como fator desencadeador de punição ou reforçamento ao comportamento dos filhos) e de disciplina relaxada (não cumprimento de regras ou rotinas pré-estabelecidas). Ainda que não com as mesmas definições estabelecidas por classes de respostas como fez Gomide (2006) ao descrever os dois conjuntos de práticas positivas e os cinco de práticas negativas, outros estudos têm apontado que comportamentos utilizados pelas mães podem levar ao desenvolvimento de comportamentos inadequados dos bebês. Esses comportamentos maternos podem envolver o uso da hipervigilância, intrusividade, baixa sensibilidade aos filhos ou pouca estimulação cognitiva, tal como descrito nos estudos de Farmer e Lee (2012), Kaitz et al. (2010), Pereira et al. (2012) e Wijnroks (1999).

Considerando os resultados obtidos e o modelo de práticas educativas parentais para díades mãe-bebê, tornase importante retomar, especialmente, a conceituação e exemplificação das práticas negativas de punição inconsistente e disciplina relaxada em se tratando de bebês em tenra idade. Assim, ao responder o instrumento utilizado para a identificação de práticas de punição inconsistente, as mães deveriam assinalar a frequência com que emitiam os comportamentos descritos pelas seguintes questões: "quando meu filho faz algo que me desagrada, o modo como respondo a ele depende do meu humor", "quando estou alegre, não me importo com comportamentos do meu filho que me desagradam", "trato mal meu filho quando estou nervosa, mas assim que a raiva passa, me arrependo", "quando estou nervosa, desconto no meu filho" e "sou mal- humorada com meu filho".

Da mesma forma, para a identificação das práticas de disciplina relaxada, as participantes deveriam assinalar a frequência com que emitiam os comportamentos descritos pelas seguintes questões: "ameaço que vou bater, mas depois não faço nada", "estabeleço uma rotina, mas nunca consigo segui-la", "se meu filho chora digo que não vou pegá-lo no colo mas, se ele insiste em chorar, acabo pegando", "Não faço horários para meu filho, as coisas acontecem naturalmente" e "aviso que não vou pegar no colo quando meu filho faz birra, mas depois acabo ficando com pena e pego no colo".

De forma semelhante, o estudo de Altafim (2012), que também utilizou o IEPMB para a identificação de práticas educativas de mães de bebês, verificou o uso de práticas de punição inconsistente e disciplina relaxada. Ainda que a literatura cite práticas de caráter disciplinador a partir dos 12 meses e enfatize os anos pré-escolares (Marin, Piccinini, \& Tudge, 2011, Boas \& Bolsoni-Silva, 2010), entende-se que há a necessidade de identificá-las em momentos anteriores do desenvolvimento infantil, o que se mostra escasso na literatura nacional.

Considerando os resultados do presente estudo, notouse que, quando comparados os grupos de cada indicador emocional em termos de presença/ausência, observou-se que, para três dos quatro indicadores (estresse, ansiedade estado e depressão) os grupos com e sem eram equivalentes e as práticas educativas negativas destacadas foram punição inconsistente para ansiedade-estado e depressão, bem como disciplina relaxada para estresse. Bekkhus et al. (2011), ainda que relacionassem ansiedade e depressão aos comportamentos de choro e de agressão física das crianças do seu estudo, não se referiram a que práticas educativas maternas esses indicadores estariam associados.

No que se refere à ansiedade-traço, os grupos não eram equivalentes em escolaridade, que era mais baixa para o grupo com ansiedade. Os grupos apresentaram diferenças significativas em disciplina relaxada e em punição inconsistente, com frequência maior para o Grupo com ansiedade. A escolaridade é uma variável sociodemográfica importante que pode ser um fator de risco para o aparecimento de indicadores emocionais. Belsky (1984) já chamava a atenção para o fato de que as práticas educativas parentais são influenciadas pelos indicadores emocionais maternos e estes pelos indicadores sociodemográficos. Assim, o choro do bebê pode ser um estímulo aversivo, bem como o fato de o bebê ficar doente ou se machucar ou, em último caso, de a mãe não ter condições materiais de prover as necessidades de seu filho, em situações de pobreza. Esses são estímulos aversivos relacionados ao bebê que podem influenciar as respostas ansiosas maternas, mas, além deles, há que se supor a existência de situações relacionadas ao trabalho ou à vida social das mães que podem também conter elementos de aversividade.

Schwengber e Piccinini (2005) colocam o manejo do bebê como um fator possível de dificuldades das mães. $\mathrm{O}$ presente estudo ressalta a importância das práticas educativas parentais para o cuidado com os bebês, pois, uma vez que as mães tenham o repertório comportamental parental repleto de práticas positivas e poucas negativas, o manejo do filho pode ser mais funcional e, portanto, menos estressante.

Para uma outra análise da influência dos indicadores emocionais sobre as práticas educativas maternas, considerouse a possibilidade de que, quanto mais indicadores, piores as práticas. Grupos foram organizados considerando a presença de todos os indicadores, de dois e três indicadores e de um indicador, comparando-os com o grupo com nenhum indicador. Os dados apontaram que o Grupo 2 era equivalente ao Grupo $1 \mathrm{em}$ idade e escolaridade, também não se observando diferenças entre as práticas relatadas pelos seus componentes. Os Grupos 1 e 3 também eram equivalentes em idade e escolaridade, mas significativamente diferentes no uso da prática negativa punição inconsistente, mais frequente entre as participantes do Grupo 3. Os Grupos 1 e 4 eram equivalentes em idade, mas não em escolaridade. Novamente há a possibilidade de que essas variáveis sociodemográficas tenham uma interferência positiva na presença dos indicadores e, também, nas práticas negativas. Altafim (2012) encontrou correlação negativa significativa entre escolaridade materna e as práticas negativas de disciplina relaxada e punição inconsistente, presentes nas mães com escolaridade menor.

Em suma, entendendo que a parentalidade é multideterminada (Belsky, 1984), um dos fatores determinantes pode ser a saúde mental materna, também influenciada por variáveis sociodemográficas. O importante é estudá-las amplamente, investindo na interação saudável das mães com seus filhos e proporcionando um ambiente minimamente adequado para o desenvolvimento infantil. 
Nesse sentido, pode ser oportuno intervir junto às mães, ensinando-lhes comportamentos alternativos, que ampliem seu repertório comportamental. No caso específico de bebês, pode ser necessário ajudar as mães a se comportarem diante das necessidades básicas e fisiológicas de seus filhos, bem como ajudá-las a demonstrar afeto, promovendo uma interação adequada entre mãe e bebê.

\section{Considerações Finais}

Os resultados obtidos na presente pesquisa apontam que, pelo menos duas práticas educativas negativas, a disciplina relaxada e a punição inconsistente, estão mais presentes em mães de bebês com qualquer um dos indicadores emocionais avaliados (estresse, ansiedade-estado, ansiedade-traço e depressão). Ainda que, em duas situações, os grupos não tenham se mostrado homogêneos com relação à escolaridade, pelo menos um estudo (Altafim, 2012) identificou as mesmas práticas relacionadas à baixa escolaridade materna. Tais dados reforçam a necessidade de se observar a saúde mental materna e elaborar programas que atendam essas mães, tanto em relação à saúde mental como às práticas utilizadas com seus filhos.

Os resultados ora apresentados contribuem para a expansão de pesquisas nacionais que se referem a práticas educativas de mães de bebês em tenra idade, associando variáveis maternas de saúde mental aos comportamentos parentais. Identificamos a necessidade de estudos que analisem o peso de todas as variáveis possíveis de forma a determinar o nível de relação entre elas. Outra questão relevante a ser explorada nos próximos estudos refere-se às crenças maternas, que são também comportamentos que podem estimular as mães a agirem de determinadas formas com seus filhos.

$\mathrm{Na}$ presente pesquisa, o autorrelato das participantes foi priorizado. Há, na literatura, estudos que também utilizaram esse método (Bolsoni-Silva \& Loureiro, 2010; Smith, 2012). Assim, ainda que a observação direta não tenha sido realizada na presente pesquisa, a utilização de instrumentos permitiu hipotetizar relações entre o organismo e o ambiente (Leme, Bolsoni-Silva, \& Carrara, 2009). Pesquisas futuras podem contemplar, em seu método, situações de observação direta, conforme sugerido por Macarini, Martins, Minetto e Vieira (2010), que mencionam que os estudos com pais de crianças pequenas é delicado porque depende somente do relato dos pais.

O estudo das práticas educativas parentais, mais frequente entre pais e mães de crianças a partir de um ano, também é possível e útil desde o nascimento. O fortalecimento de práticas educativas positivas, com certeza, minimiza o aparecimento das práticas educativas negativas, prejudiciais para a interação mãe-filho. Estudos que identifiquem tipos de práticas educativas tais como definidas por Gomide (2006) facilitam a elaboração de programas de intervenção pontuais para mães de crianças que considerem a idade dos filhos, auxiliando-as nas reflexões acerca dos desdobramentos de suas ações sobre o comportamento da criança. Intervenções precoces podem promover interações mais prazerosas entre pais e filhos de qualquer idade, além de resultar no seu desenvolvimento sadio.

\section{Referências}

Altafim, E. R. P. (2012). Práticas parentais de mães de bebês: A influência de variáveis maternas e do bebê (Unpublished marster's thesis). Programa de Pós-Graduação em Psicologia do Desenvolvimento e Aprendizagem, Universidade Estadual Paulista "Júlio de Mesquita Filho", Bauru, SP.

Altafim, E. R. P., Schiavo, R. A., \& Rodrigues, O. M. P. R. (2008). Práticas parentais de mães adolescentes: um estudo exploratório. Temas sobre Desenvolvimento, 16, 104-110.

Bagner, D. M., Pettit, J. W., Lewinsohn, P. M., \& Seeley, J. R. (2010). Effect of maternal depression on child behavior: a sensitive period? Journal of the American Academy of Child e Adolescent Psychiatry, 49(7), 699- 707.

Banaco, R. (2005, Agosto). Stress e Terapia Comportamental. In XIV Encontro Brasileiro de Psicoterapia e Medicina Comportamental. Campinas, SP.

Baptista, M. N., Baptista, A. S. D., \& Torres, E.C.R. (2006). Associação entre suporte social, depressão e ansiedade em gestantes. PSIC-Revista de Psicologia da Vetor Editora, 7(1), 39-48.

Bekkhus, M., Rutter, M., Barker, E. D., \& Borge, A. I. H. (2011). The role of pre- and postnatal timing os family risk factors on child behavior at 36 months. Journal Abnormal Child Psychology, 39, 611- 621 .

Belsky, J. (1984). The determinants of parenting: A process model. Child Development, 55(1), 83-96.

Boas, A. C. V. B. V., \& Bolsoni- Silva, A. T. Habilidades sociais educativas de mães separadas e sua relação com o comportamento de pré- escolares. Psico- USF, 15(3), 301-310.

Bolsoni-Silva, A. T., \& Loureiro, S. R. (2010). Validação do roteiro de entrevista de habilidades sociais educativas parentais (REHSE-P). Avaliação Psicológica, 9(10), 63-75.

Bolsoni-Silva, A. T., \& Marturano, E. M. (2007). A qualidade da interação positiva e da consistência parental na sua relação com problemas de comportamentos de pré-escolares. Interamerican Journal of Psychology, 41, 349-358.

Camelo, S. H. H, \& Angerami, E. L.S. (2004). Sintomas de estresse nos trabalhadores atuantes em cinco núcleos de saúde da família. Revista Latino-Americana de Enfermagem, Ribeirão Preto, 12(1), 14-21.

Correia, L. L., \& Linhares, M. B. M. (2007). Ansiedade materna nos períodos pré e pós-natal: revisão da literatura. Revista Latino-Americana de Enfermagem, 15(4), 677-683.

Cury, A. F., \& Menezes, P. R. (2006). Ansiedade no puerpério: Prevalência e fatores de risco. Revista Brasileira de Ginecologia e Obstetrícia, 28(3), 171 -178.

Dancey, C. P., \& Reidy, J. (2006). Estatística sem matemática para Psicologia. Porto Alegre: Artmed.

Farmer, A. Y., \& Lee, S. K. (2011). The Effects of Parenting Stress, Perceived Mastery, and Maternal Depression on Parent-Child Interaction. Journal of Social Service Research, 37(5), 516525 . 
Ferreira, M., \& Marturano, E.M. (2002). Ambiente familiar e os problemas de comportamento apresentados por crianças com baixo desempenho escolar. Psicologia: Reflexão e Crítica, 15(1), 35-44.

Ferster, C. B., Culbertson, S., \& Perrot-Boren, M. C. (1977). Depressão Clínica. In C. B. Ferster, S. Culbertson \& M. C. Perrot-Boren (Org.), Princípios do Comportamento (pp. 699725). São Paulo: Hucitec.

Field, T. (1995). Infants of depressed mothers. Infant Behavior and Development, 18, 1-13.

Field, T. (1992). Infants of depressed mothers. Development and Psychopathology, 4, 49-66.

Fioravanti, A. C. M., Santos, L. F., Maissonette, S., Cruz, A. P. M., \& Landeira-Fernandez, J. (2006). Avaliação da estrutura fatorial da Escala de Ansiedade-Traço do IDATE. Avaliação Psicológica, 5(2), 217-224.

Fraga, D. A., Linhares, M. B. M., Carvalho, A. E. V., \& Martinez, F. E. (2008). Desenvolvimento de bebês nascidos pré-termo e indicadores emocionais maternos. Psicologia: Reflexão $e$ Crítica, 21(1), 33-41.

Gomes, V. F., \& Bosa, C. (2004). Estresse e relações familiares na perspectiva de irmãos de indivíduos com Transtornos Globais do Desenvolvimento. Estudos de Psicologia, 9(3), 553- 561.

Gomide, P. I. C. (2006). Inventários de Estilos Parentais (IEP): Modelo teórico, manual de aplicação, apuração $e$ interpretação. Rio de Janeiro: Editora Vozes.

Gomide, P. I. C., Salvo, C. G., Pinheiro, D., \& Sabbag, G. (2005). Correlação entre práticas educativas, depressão, estresse e habilidades sociais. Revista Psico-USF, 10(2), 169- 178.

Gongora, M.A.N. (1981). Conceitos de depressão. Semina, 7(2), 115-120.

Gorestein, C., Wang, W. Y., Argimon, I. L., \& Werlang, B. S. G. (2011). Manual do Inventário de Depressão de Beck- BDI-II ( $2^{\text {a }}$ ed). São Paulo: Casa do Psicólogo.

Goulart, E. Jr., \& Lipp, M. E. N. (2008). Stress entre professoras do ensino fundamental de escolas públicas estaduais. Psicologia em Estudo, 13(4), 847-857.

Kaitz, M., Maytal, H. R., Devor, N., Bergman, L., \& Mankuta, D. (2010). Maternal anxiety, mother- infant interactions, and infants' response to challenge. Infant Behavior \& Development, 33, 136-148.

Kazdin, A. E., \& Whitley, M. K. (2003). Treatment of parental stress to enhance therapeutic change among children referred for aggressive and antisocial behavior. Journal of consulting and clinical psychology, 71(3), 504- 515.

Leme, V. B. R., Bolsoni-Silva, A. T., \& Carrara, K. (2009). Uma análise comportamentalista de relatos verbais e práticas educativas parentais: Alcance e limites. Paideia, 19(43), 239-247.

Letourneau, N. L., Tramonte, L., \& Willms, D. (2013). Maternal depression, family functioning and children's longitudinal development. Journal of Pediatric Nursing 28(3), 223-234.

Lipp, M. E. N. (2000). Manual do Inventário de Sintomas de Stress para Adultos de Lipp (ISSL). São Paulo: Casa do Psicólogo.

Macarini, S. M., Martins, G. D. F., Minetto, M. F. J, \& Vieira, M. L. (2010). Práticas parentais: Uma revisão da literatura brasileira. Arquivos Brasileiros de Psicologia, 62(1), 119-134.
Manuel, J. I., Martinson, M. L., Bledsoe-Mansori, S. E., \& Bellamy, J. L. (2012). The influence of stress support on depressive symptoms in mothers with young children. Social Science \& Medicine, 75, 2013- 2020.

Marin, A. H., Piccinini, C. A., \& Tudge, J. R. H. (2011). Estabilidade e mudança nas práticas educativas maternas e paternas ao longo dos anos pré-escolares da criança. Psicologia: Reflexão e Crítica, 24(10), 71-79.

Nogueira, S. C., Rodrigues, O. M. P. R., \& Altafim, E. R. P. (2013). Práticas educativas de mães de bebês: Efeitos de um programa de intervenção. Psicologia em Estudo, 18(4), 599-609.

Padovani, F. H. P., Linhares, M. B. M., Carvalho, A. E. V., Duarte, G., \& Martinez, F. E. (2004). Avaliação de sintomas de ansiedade e depressão em mães de neonatos pré-termo durante e após hospitalização em UTI-Neonatal. Revista Brasileira de Psiquiatria, 26(4), 251-4.

Paranhos, M. E., Argimon, I. I. L., \& Werlang, B. S. G. (2010). Propriedades psicométricas do Inventário de Depressão de Beck-II (BDI-II) em adolescentes. Avaliação Psicológica, 9(3), 383-392.

Patterson, G., Reid, J., \& Dishion, T. (2002). Antisocial boys. Eugene: Castalia.

Pereira, J., Vickers, K., Atkinson, L., Gonzales, A., Wekerle, C., \& Levitan, R. (2012). Parenting stress mediates between maternal maltreatment history and maternal sensitivity in a community sample. Child Abuse \& Neglect, 36, 433- 437.

Perosa, G. B., Canavez, I. C., Silveira, F. C. P., Padovani, F. H. P., \& Peraçoli, J. C. (2009). Sintomas depressivos e ansiosos em mães de recém-nascidos com e sem malformações. Revista Brasileira de Ginecologia e Obstetrícia, 31(9), 433- 439.

Perosa, G. B., Silveira, F. C. P., \& Canavez, I. C. (2008). Ansiedade e depressão de mães de recém- nascidos com malformações visíveis. Psicologia: Teoria e Pesquisa, 24(1), 029-036.

Resolução $n^{\circ} 466$, de 12 de dezembro de 2012 (2012). Dispõe sobre pesquisas com seres humanos. Conselho Nacional de Saúde - CNS. Recuperado em 27 de fevereiro de 2015, de <http:// conselho.saude.gov.br/resolucoes/2012/Reso466.pdf >

Rodrigues, O. M. P. R., Altafim, E. R. P., \& Schiavo, R. A. (2011). Práticas parentais de mães adultas e adolescentes com bebês de um a doze meses. Aletheia, 34, 96-108.

Rodrigues, O. M. P. R., Nogueira, S. C., \& Altafim, E. R. P. (2013). Práticas parentais maternas e a influência de variáveis familiares e do bebê. Pensando Famílias, 17(2), 71-83.

Salvo, C. G., Silvares, E. F. M., \& Toni, P. M. (2005). Práticas educativas como forma de predição de problemas de comportamento e competência social. Estudos de Psicologia/ Campinas, 22(2), 187- 195.

Schiavo, R. A. (2011). Presença de stress e ansiedade em primigestas no terceiro trimestre de gestação e no pós-parto (Unpublished master's thesis). Programa de Pós-Graduação em Psicologia do Desenvolvimento e Aprendizagem, Universidade Estadual Paulista "Júlio de Mesquita Filho", Bauru, SP.

Schwengber, D. D. S., \& Piccinini, C.A. (2005). A experiência da maternidade no contexto da depressão materna no final do primeiro ano de vida do bebê. Estudos de Psicologia, 22(2), 143-156.

Selye, H. (1956). The stress of life. New York: Mc Graw Hill. 
Smith, S. M. (2012). Do parenting practices mediate the relation between maternal depression and child adaptative behaviors? An examination of outcomes for children from varying socioeconomic status groups. Trabalho de Conclusão de Curso, Department of Psychology, University of Southern Mississippi, Hattiesburg, MS.
Spielberger, C. D, Gorsuch, R. L, \& Lushene, R. E. (2003). Inventário de ansiedade traço-estado - IDATE ( $2^{\mathrm{a}}$ ed., A. Biaggio, Trad \& Adapt.). Rio de Janeiro: CEPA.

Wijnroks, L. (1999). Maternal recollected anxiety and mother infant interaction in preterm infants. Infant Mental Health Journal, 20(4), 393-409.

Zamignani, D. R., \& Banaco, R. A. (2005). Um Panorama AnalíticoComportamental sobre os Transtornos de Ansiedade. Revista Brasileira de Terapia Comportamental e Cognitiva, VI(1), 077-092. 